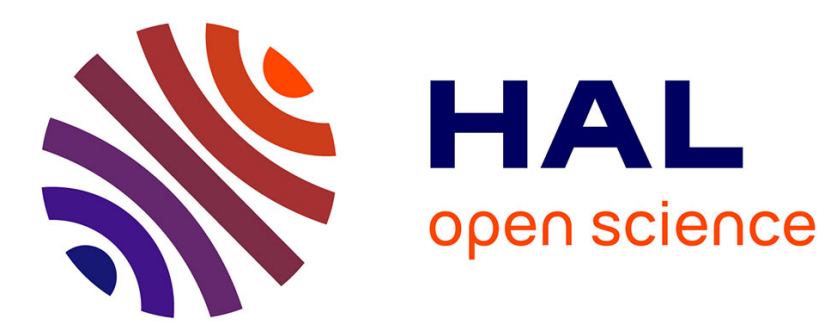

\title{
Des sites sans visiteurs: les mémoriaux du camp de Salaspils et de la forêt de Bikernieki en Lettonie
}

\author{
Fabrice Virgili
}

\section{To cite this version:}

Fabrice Virgili. Des sites sans visiteurs : les mémoriaux du camp de Salaspils et de la forêt de Bikernieki en Lettonie. Les Cahiers Sirice / Irice , 2011, 7, pp.101-110. hal-00687371

\section{HAL Id: hal-00687371 \\ https://hal.science/hal-00687371}

Submitted on 13 Apr 2012

HAL is a multi-disciplinary open access archive for the deposit and dissemination of scientific research documents, whether they are published or not. The documents may come from teaching and research institutions in France or abroad, or from public or private research centers.
L'archive ouverte pluridisciplinaire HAL, est destinée au dépôt et à la diffusion de documents scientifiques de niveau recherche, publiés ou non, émanant des établissements d'enseignement et de recherche français ou étrangers, des laboratoires publics ou privés. 


\title{
DES SITES SANS VISITEURS : LES MÉMORIAUX DU CAMP DE SALASPILS ET DE LA FORÊT DE BIKERNIEKI EN LETTONIE
}

\author{
Fabrice Virgili
}

IRICE | Les cahiers Irice

\author{
2011/1 - $\mathrm{n}^{\circ} 7$ \\ pages 101 à 110
}

ISSN 1967-2713

Article disponible en ligne à l'adresse:

http://www.cairn.info/revue-les-cahiers-irice-2011-1-page-101.htm

Pour citer cet article

Virgili Fabrice, « Des sites sans visiteurs : les mémoriaux du camp de Salaspils et de la forêt de Bikernieki en Lettonie ",

Les cahiers Irice, 2011/1 n7, p. 101-110.

Distribution électronique Cairn.info pour IRICE.

(c) IRICE. Tous droits réservés pour tous pays.

La reproduction ou représentation de cet article, notamment par photocopie, n'est autorisée que dans les limites des conditions générales d'utilisation du site ou, le cas échéant, des conditions générales de la licence souscrite par votre établissement. Toute autre reproduction ou représentation, en tout ou partie, sous quelque forme et de quelque manière que ce soit, est interdite sauf accord préalable et écrit de l'éditeur, en dehors des cas prévus par la législation en vigueur en France. II est précisé que son stockage dans une base de données est également interdit. 


\section{Des sites sans visiteurs : \\ les mémoriaux du camp de Salaspils \\ et de la forêt de Bikernieki en Lettonie}

Fabrice VIRGILI

Ceux qui se rendent aujourd'hui à Auschwitz, au-delà de ce qu'ils peuvent ressentir ou apprendre, constatent aussi qu'ils sont loin d'être les seuls à visiter ce symbole de l'extermination nazie. L'évolution du nombre de visiteurs confirme le caractère exceptionnel pris par ce site. En dix ans le nombre de visites annuelles est passé de 500000 à 1300000 . Dans le même temps, d'autres lieux connus de l'univers concentrationnaire ont vu leur fréquentation stagner, tout en demeurant au-dessus de plusieurs dizaines de milliers de visiteurs (jusqu'à 400000 pour Sachsenhausen au nord de Berlin). À l'inverse, d'autres sites se visitent seuls ou presque, à l'image de deux mémoriaux proches de Riga en Lettonie, le mémorial de Salaspils et celui de la forêt de Bikernieki ${ }^{1}$. Je les ai visités comme touriste, sans projet scientifique et sans savoir à l'époque que j'allais en faire part à l'occasion de cette journée. Je ne suis un spécialiste ni de l'histoire de la Shoah, ni de celle des pays baltes pendant le Second conflit mondial ; j'ai visité ces lieux sans avoir rien lu, et sans en avoir entendu parler auparavant, décidant de m'y rendre à la simple lecture du Guide du routard.

Le sentiment de solitude commence avec la recherche du lieu : "Le mémorial de $1^{\prime}$ holocauste de la forêt de Bikernieku (hors plan I) Bikernieki iela, vers le village de Mezciems. Pour s'y rendre, bus $n^{\circ} 15$, 16, 31, arrêt Kapi, puis $200 \mathrm{~m}$ à pied par un petit chemin ; sinon trolleys $\mathrm{n}^{\circ} 14,18$, arrêt Keguma, puis $1 \mathrm{~km}$ de marche à pied dans la forêt, sur Bikernieku (puis chemin pour le mémorial à droite bien indiqué) $»^{2}$. Il est en effet bien indiqué une fois que vous l'avez trouvé, bien qu'auparavant

1 Il existe un troisième site important à proximité de Riga, celui de la forêt de Rumbula où furent abattus 25000 Juifs au début du mois de décembre 1941.

2 Le guide du routard, Pologne et capitales baltes 2009/2010, Paris, Hachette, 2009, p. 479. 
rien ne vous oriente vers l'endroit que vous recherchez. Quelques places de stationnement vides à l'entrée du chemin, une prostituée qui déambule à proximité. Vous pénétrez alors dans la forêt pour vous trouver en présence de quelques poteaux de béton, qui délimitent le site, sur lesquels sont gravés, ici une étoile de David, là une croix chrétienne, plus loin des lauriers (et non une faucille et un marteau) évoquant les prisonniers de guerre soviétiques ou des communistes lettons également abattus à cet endroit. Au centre, la clairière où a été érigé le mémorial. Une arche protège un cube de marbre noir sur lequel est gravé sur chacune des faces le vers $16: 18$ du livre de Job, en hébreux, en letton, en allemand : «Ô terre, ne couvre point mon sang, Et que mes cris prennent librement leur essor !».

Tout autour des pierres plantées dans le sol - qui ne sont pas sans évoquer le mémorial bien antérieur de Treblinka et les longs panoramiques qu'en fit Claude Lanzmann dans Shoah - divisées selon les villes d'où vinrent les personnes exécutées : Lubeck, Berlin, d'autres villes allemandes et autrichiennes, mais aussi Riga. La forêt de Bikernieki fut en effet avec Rumbula, un des principaux lieux de massacre des Juifs lettons, mais aussi allemands déportés dans le ghetto de Riga avant d'être assassinés. Berlin, gravé au sol, fait écho aux « Riga » qui figurent sur les quais du mémorial Gleis 17 de la gare de Grunewald à Berlin. C'est en effet de là que partirent plusieurs convois emportant les Juifs berlinois vers le ghetto de Riga puis dans les lieux de mises à mort environnants.

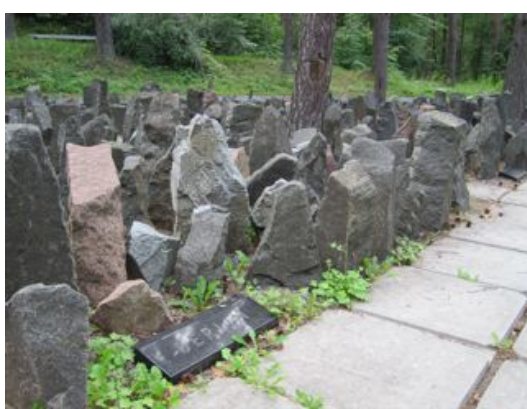

Bikernieki : Indication des lieux d'origine des personnes exécutées

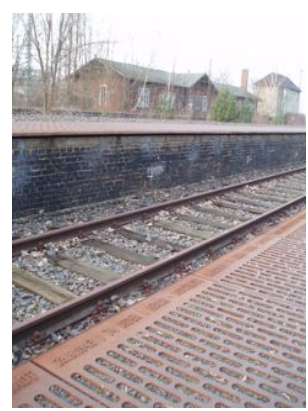

Grunewald : indication des destinations des personnes déportées ${ }^{3}$

3 Toutes les photographies ont été réalisées par l'auteur à l'occasion d'un voyage à Berlin en mars 2008 et en Lettonie en août 2009. 


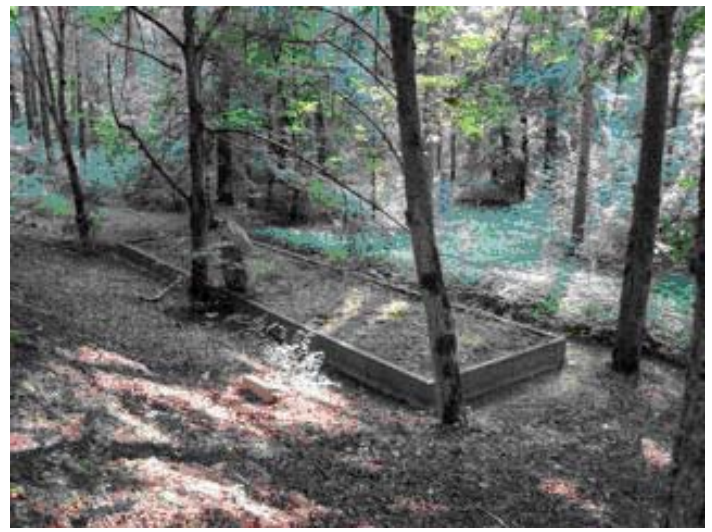

Une trentaine de rectangles signalent à travers les arbres les fosses qui ont été retrouvées. Nous sommes donc dans un cimetière, où les corps n'ont pas été réduits en cendres mais se trouvent quelques mètres sous terre. Pas de musée, ni de boutique où seraient vendus ouvrages et brochures sur le sujet, seules d'immenses tombes éparses dans les sous-bois. Non par choix, comme le sont souvent dans cette partie boisée de l'Europe les cimetières, mais parce que l'inhumation se confond ici avec l'exécution. Les chiffres varient selon les auteurs mais le nombre de victimes se compte en dizaines de milliers.

Un lieu vide pourtant, non de sens, mais de visiteurs: quelques cailloux déposés témoignent de rares passages et moments de recueillement et si l'on croise quelques personnes, ce sont des habitués, joggeurs à travers les bois ou couples de jeunes amoureux fumant tranquillement une cigarette sur un banc à proximité. Au sol, quelques canettes de bière et tessons de bouteille témoignent encore d'une fréquentation ignorante du site.

Sentiments similaires aux abords de Salaspils ${ }^{4}$. À une vingtaine de kilomètres au sud-est de Riga, une seule indication à l'approche du site et plusieurs bifurcations, un cul-de-sac où stationne un poids lourd et à nouveau une prostituée, habituée manifeste d'un lieu tranquille.

4 Pour en savoir plus sur le camp de Salaspils voir: Andrej Angrick, Peter Klein, The "Final Solution" in Riga: Exploitation and Annihilation, 1941-1944, Londres, Berghahn books, 2009. 


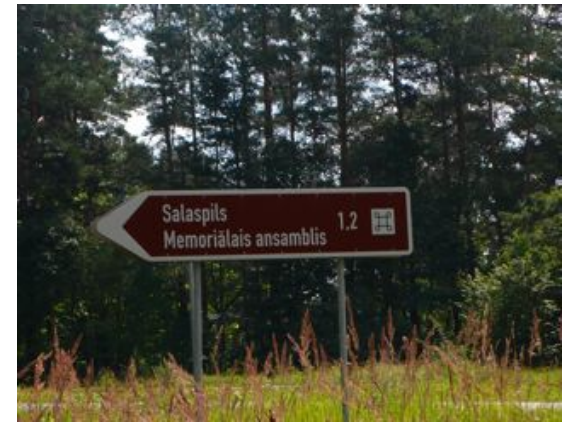

L'unique panneau indicateur

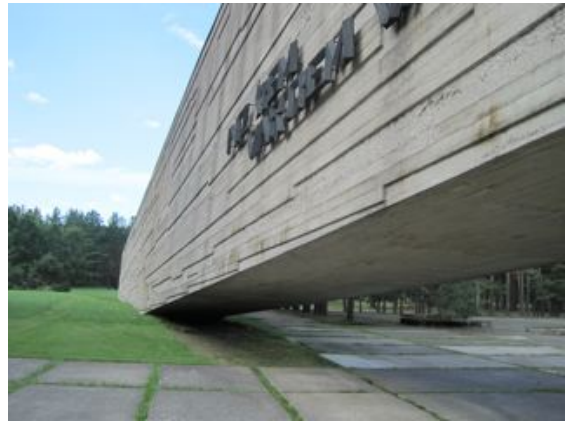

L'entrée du site

Là encore, la forêt fait écran, choisie à dessein par les Allemands pour $\mathrm{y}$ masquer camps et/ou lieux de mise à mort, elle continue à masquer encore aujourd'hui ces sites. Pourtant celui de Salaspils est très étendu : une immense clairière à laquelle on accède après avoir franchi un mur incliné de béton portant l'inscription «Derrière cette porte la terre pleure ». Sentiment de solitude démultiplié par l'ampleur du mémorial et l'absence totale de visiteurs pourtant en plein mois d'août, le pic de fréquentation pendant ce temps à Auschwitz où une personne passe toutes les deux secondes sous la porte Arbeit macht Frei ${ }^{5}$. De l'ancien camp il ne reste rien, si ce n'est le tracé reconstitué au sol de la place centrale du camp et de quelques baraquements. Ici, comme dans de nombreux autres lieux du système concentrationnaire nazi, les bourreaux prirent soin de détruire les traces de leur crime ; les constructions furent brûlées ${ }^{6}$, les archives emportées ou détruites au cours de l'été 1944 à l'approche de l'Armée rouge. Ce fut en octobre 1967, pendant la période soviétique, que le mémorial fut inauguré. Ses architectes et sculpteurs furent récompensés pour leur œuvre par le prix Lénine en 1970. L'intention était à la fois pédagogique, une modeste exposition permanente figurait à l'intérieur du «mur » de béton, et politique, ériger un tel monument en République socialiste soviétique de Lettonie était un moyen de dénoncer en creux l'engagement de nombreux Lettons auprès des nazis et de légitimer le rôle de l'Armée rouge. Depuis l'indépendance

5 Voir dans le même volume Piotr Cywinski, "Auschwitz, site mémoriel au XXI siècle $»$, p. 9.

6 Céline Bayou et Éric Le Bourhis, « Rendez-vous manqué entre histoire et mémoire. Le camp de concentration de Salaspils en Lettonie », Le Courrier des pays de l'Est, 2007/6, $\mathrm{n}^{\circ} 1064$, p. 69. 
des pays baltes en 1991, la mémoire de la Seconde Guerre mondiale et son articulation avec la période soviétique est particulièrement sensible. Les productions mémorielles nationales récentes tendent à estomper, si ce n'est effacer, le souvenir de l'occupation nazie pour mettre en avant l'horreur de la domination soviétique. En Lettonie, où la minorité russophone est la plus importante, le récit prend une dimension polémique et politique accrue, à l'intérieur du pays comme vis-à-vis de la Russie voisine. Les débats concernent aussi bien l'origine des victimes, leur nombre respectif, le rôle des auxiliaires lettons, que le financement, la dédicace et l'usage du mémorial7.

Rien de tout ça n'apparaît lors de la visite, excepté la marque de l'époque soviétique dont les statues colossales sont le plus évident témoignage. Sur la gauche $\mathrm{du}$ site, deux figures de femmes, «l'Humiliée » et «la Mère ». On retrouve ici deux images traditionnelles de la propagande de guerre soviétique ${ }^{8}$. La posture agenouillée, le bras venant masquer le bas du visage et le regard vers le sol, toute la sculpture désigne une image féminine de la honte, celle de la femme victime d'un viol, que l'on retrouve sur de nombreuses affiches en 19411945. Elle est le double symbole de l'invasion du pays et de la barbarie nazie. Quelques dizaines de mètres plus loin, on retrouve "La Mère patrie», abondamment utilisée pour la mobilisation de guerre, elle contraste par son regard et sa posture avec la femme précédente. Seule la femme dans son rôle maternel et protecteur est valorisée, d'autant qu'ici furent détenus et moururent plusieurs centaines, voire des milliers d'enfants. Non loin de l'emplacement de la statue se trouvaient les baraques qu'ils occupaient; à l'emplacement de l'une d'entre elles un monument sur lequel ont été déposés peluches et jouets.

7 Voir à ce propos l'article de Céline Bayou et Éric Le Bourhis, «Rendez-vous manqué entre histoire et mémoire...», op. cit., p. 65-76, qui propose une excellente mise au point à ce sujet.

8 Christophe Barthélémy, "Vierges et martyres dans l'imagerie soviétique ", dans François Rouquet, Fabrice Virgili, Danièle Voldman (dir.), Amours, guerres et sexualité 1914-1945, Paris, Gallimard, 2007, p. 99-101. 


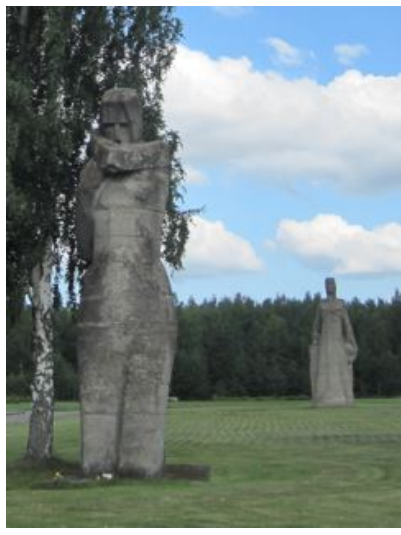

L'humiliée

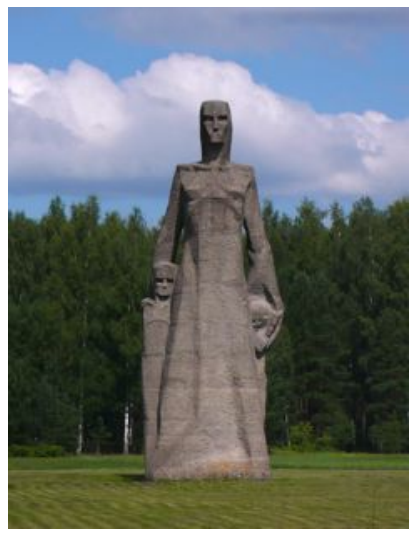

La Mère

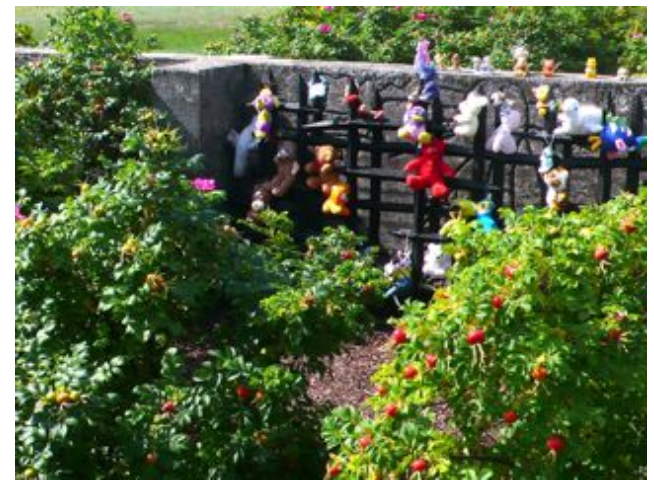

Baraquement des enfants

Du côté droit sont érigées les statues masculines: "L'homme qui résiste ${ }^{9}$ isolé comme «l'Humiliée », mais à la différence de celle-ci, le sculpteur le fait tenir malgré la souffrance car «les personnes éprises de liberté ne peuvent jamais être anéanties $»^{10}$. Les trois autres sont «Solidarité », « le Serment », et « Front rouge». Ils font groupe et rien ne saurait briser ces hommes soutenus par la foi en leur engagement, tous derrière l'homme le point levé.

9 Les titres proposés sont en letton « Nesalautztais », en anglais « The Unbroken » et en allemand « Der Unbeugsame».

10 A. Gavers, T. Vilemsons, Salaspils, Riga, Avots, 1989. 


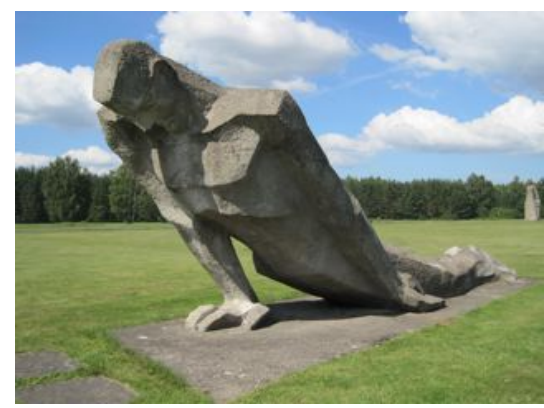

L'homme qui résiste
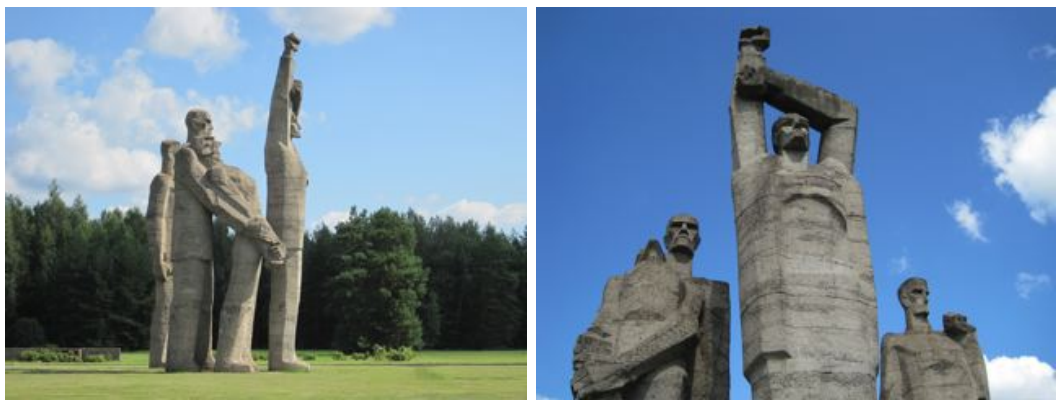

Ensemble statuaire : Solidarité, le Serment, Front rouge.

Écrasé par la taille de ces statues, avec la forêt comme seul horizon, le sentiment visuel de l'isolement est accentué par une sourde pulsation sonore qui envahit tout le site. D'un rectangle de granit noir, prévu pour recevoir les couronnes de fleurs d'éventuelles cérémonies, bat un métronome «le battement du cœur des victimes provenant des profondeurs de la terre et rappelant aux vivants : Souvenez-vous! Plus jamais ça !»11

Ce n'est qu'après un long moment passé au mémorial que vous apercevez quelqu'un, une jardinière entretenant des bosquets fleuris. À votre vue, elle sort d'un simple sac en plastique deux brochures consacrées au camp de Salaspils qu'elle vous donne sans explications. Elles sont toutes deux écrites en quatre langues (letton, russe, allemand, anglais), la première, qui date de 1989, évoque le camp construit par les «envahisseurs fascistes allemands» pendant la «Grande Guerre

11 A. Gavers, T. Vilemsons, Salaspils..., op. cit. 
patriotique ». La liste des victimes est ainsi présentée : "travailleurs et paysans lettons, femmes biélorusses, enfants et vieillards, patriotes polonais, massivement des milliers de citoyens allemands, tchèques, autrichiens, néerlandais et de nombreux autres pays ». La seconde fut réalisée pour le 40e anniversaire du mémorial en 2007. Plus sommaire, elle laisse toute la place aux photographies, le récit se contente d'évoquer des milliers de personnes d'URSS et de nombreux pays européens détenues dans le camp. Plus de mention de morts pour un camp présenté alors comme de travail et de transit.

Comme celle de bien des camps, l'histoire de Salaspils est complexe. Construit à l'été 1941 à l'initiative de la police SIPO et SD et non de la SS, il n'a pas porté l'appellation de "camp de concentration » mais celle de «prison de police et camp d'éducation par le travail ». Après avoir servi de lieu de détention de Juifs allemands, il reçut les détenus politiques et de droits-communs lettons; puis à partir de 1943, il devint camp de transit avant le départ vers les camps en Allemagne de Soviétiques soupçonnés d'aide aux partisans, y compris de nombreux enfants et adolescents. Sans oublier les très nombreux prisonniers de guerre de l'Armée rouge détenus dans le Stalag voisin. Des groupes variés et variant au gré des impératifs de l'occupant. La comparaison des deux brochures témoigne d'une constante: l'absence des Juifs. Internés de l'automne 1941 à l'été 1942, date à laquelle ils furent, soit exécutés soit d'abord transférés vers le ghetto de Riga, ce sont en fait les «citoyens allemands, tchèques et autrichiens » de la brochure de 1989. Ils n'apparaissent pas davantage en 2007, tout comme les Tziganes des pays baltes également détenus à Salaspils ${ }^{12}$. Le changement de la brochure du quarantième anniversaire consacre en revanche l'atténuation des crimes nazis. Afin d'estomper le rôle des collaborateurs lettons - qui formaient l'essentiel des gardes du camp - et de renforcer la dénonciation de la terreur soviétique après guerre, la mention des morts laisse la place à celle « d'un régime strict $»^{13}$.

12 Anton Weiss-Wendt, « Extermination of the Gypsies in Estonia during World War II: Popular Images and Official Policies ", Holocaust and Genocide Studies, Volume 17, n 1, printemps 2003.

13 Brochure, Salaspils Memorials - 40 1967-2007. 


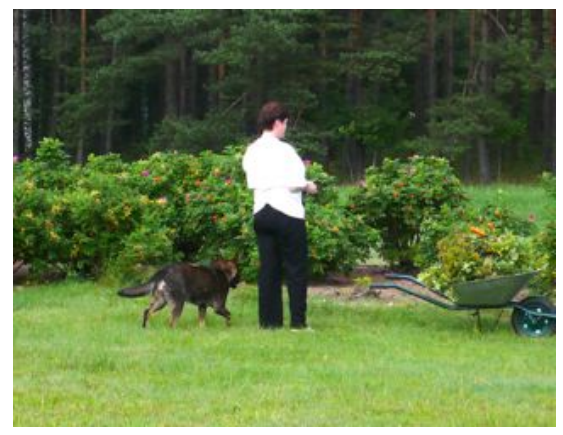

L'entretien du site
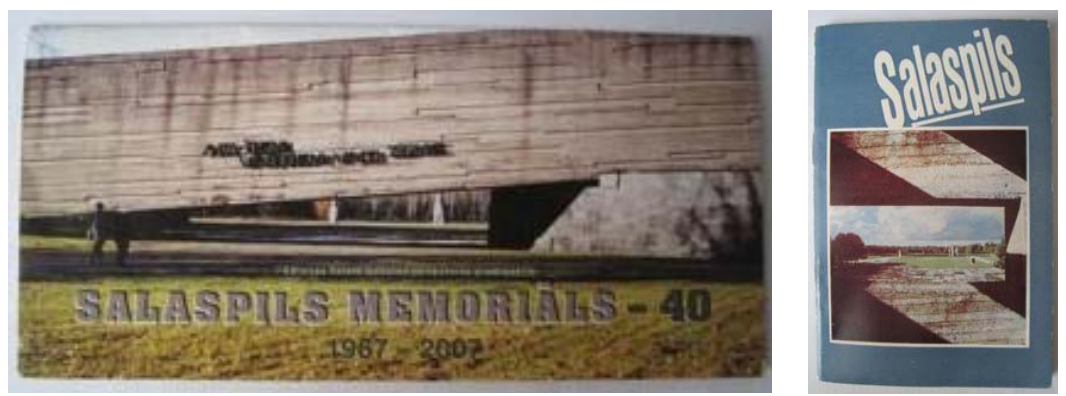

Les brochures de 1989 et de 2007

Avant de conclure cette visite, un autre point commun à Bikernieki et à Salaspils mérite d'être souligné, celui du rôle de la commission allemande des tombes de guerre (Volksbund Deutsche Kriegsgräberfürsorge) dans l'établissement et l'entretien des sites.

Bien qu'en partie détruit, le camp de Salaspils, dès que la zone fut sous le contrôle de l'Armée rouge, devint un camp de prisonniers de guerre allemands. Cent cinquante d'entre eux décédés en captivité y furent enterrés. Une fois la Lettonie indépendante, une première coopération entre la Volksbund Deutsche Kriegsgräberfürsorge et le gouvernement letton amena à l'établissement d'un premier cimetière de guerre allemand à Riga. En janvier 1996, un accord officiel s'est traduit par l'établissement d'une trentaine de cimetières de militaires allemands, mais aussi de Juifs allemands déportés et assassinés en Lettonie comme ceux de Rumbala ${ }^{14}$ et Bikernieki ${ }^{15}$. À Salaspils, le cimetière restauré en 2008 se trouve en léger retrait du camp, entouré d'un petit talus qui le

14 http://www.volksbund.de/kgs/stadt.asp?stadt=4763

15 http://www.volksbund.de/kgs/stadt.asp?stadt=4161 
soustrait pratiquement à la vue ${ }^{16}$. Le monument aux milliers de prisonniers de guerre soviétiques décédés en captivité se trouve quant à lui sur l'emplacement de l'ancien Stalag à quelques kilomètres de là.

À Bikernieki, en revanche, pas de voisinage des bourreaux et des victimes. Le monument a été érigé en 2001 par la commission allemande des tombes de guerre grâce au soutien financier du Deutsches RigaKomitee. L'association fut fondée peu de temps auparavant et regroupe les municipalités des villes dont furent déportés les Juifs vers Riga et tués ici ${ }^{17}$.

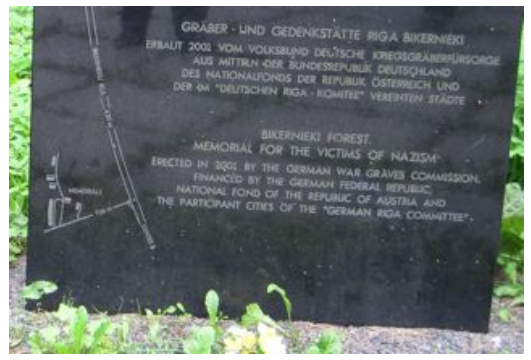

Stèle à l'entrée du site de Bikernieki

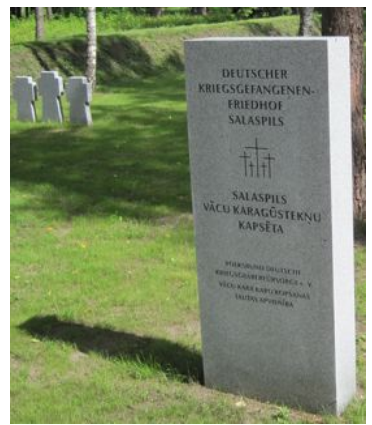

Stèle à l'entrée du cimetière militaire allemand de Salaspils

On constate donc une activité commémorative non négligeable, des enjeux politiques, symboliques et financiers, mais un désintérêt de la part des Lettons. Céline Bayou et Éric le Bourhis ${ }^{18}$ soulignent combien le mémorial de Salaspils, mais il en est de même de Bikernieki, n'est pas un lieu de «mémoire vive ». Non seulement il n'y a pas d'appropriation par les habitants, mais ces sites nuisent à une histoire plus nationaliste que nationale vingt ans après l'indépendance. La forêt, après avoir caché les crimes de l'occupation nazie, masque aujourd'hui les traces d'un passé gênant que seuls quelques visiteurs égarés ou volontaires découvrent pour l'instant.

16 http://www.volksbund.de/kgs/stadt.asp?stadt=5031

17 Ces villes sont : Berlin, Bielefeld, Dortmund, Düsseldorf, Hambourg, Hanovre, Kassel, Cologne, Leipzig, Münster, Nuremberg, Osnabrueck et Stuttgart, Bocholt, Kiel, Luebeck, Vienna, Bremen, Paderborn.

http://www.volksbund.de/schon_gelesen/spektrum/riga_english/

18 Céline Bayou et Éric Le Bourhis, «Rendez-vous manqué entre histoire et mémoire... ", art. cit., p. 75. 